\title{
Implementation of Fuzzy Localization System Based on Radio-frequency Identification Technology for Elderly People
}

\author{
Chuan-Bi Lin, Yung-Fa Huang, ${ }^{*}$ Long-Xin Chen, and Jian-Ming Hong \\ Department of Information and Communication Engineering, Chaoyang University of Technology, \\ Wufeng District, Taichung 41349, Taiwan (R.O.C.)
}

(Received August 26, 2018; accepted November 22, 2018)

Keywords: radio-frequency identification, elderly people, localization system, push notification, fuzzy logic

With the widespread development of the Internet, the Internet of Things (IoT) technology has been popularly applied to many applications, for example, network appliances, automation and control, and people position. In recent years with the aging population trend, not only the health care of elderly people but also problems associated with the loss of wisdom of the elderly has attracted increasing attention. Moreover, how to monitor elderly people has also become a very popular topic. The radio-frequency identification (RFID) technology has been developed for localization applications. However, LANDMARC, a location-sensing prototype system that uses RFID technology for locating objects inside buildings, is too expensive owing to the highly dense deployment of tags. Therefore, in this paper, a low-cost system based on RFID technology is proposed for elderly people. Furthermore, to increase the efficiency and quality of care, fuzzy logic is added to the proposed monitoring system. On the other hand, we use a time-sensitive alert marquee, email, and push notification function to give an alarm for elderly people who stay too long in the bathroom or other locations outside. The results show that caregivers can clearly monitor the positions of elderly people through the RFID-based localization system with fuzzy logic.

\section{Introduction}

The aging of the population is a worldwide trend, and developed countries are to face with this problem. Thus, many care centers or organizations composed of elderly people are being established by governments and private enterprises. However, some problems emanate from care centers such as inefficient management and missing elderly, because of shortages of software and hardware equipment. Therefore, to solve these problems, the use of Internet of Things (IoT) technology to monitor the safety and position of elderly people at care centers is widely proposed. ${ }^{(1,2)}$ In general, some sensing techniques, such as Zigbee, ${ }^{(3)}$ Bluetooth 4.0 (BLE), ${ }^{(4)}$ $\mathrm{Wi}-\mathrm{Fi},{ }^{(5)}$ radio-frequency identification (RFID), ${ }^{(6)}$ and infrared, ${ }^{(7)}$ are used to track the positions of people. However, these technologies have pros and cons for tracking positions; for example,

*Corresponding author: e-mail: yfahuang@cyut.edu.tw https://doi.org/10.18494/SAM.2019.2132 
the infrared technology has high transfer rates, but its transfer quality is easily affected by temperature changes.

Although the proposed RFID techniques, such as LANDMARC, ${ }^{(8)}$ have been used for tracking positions in indoor areas, they are too expensive owing to the highly dense deployment of tags. Thus, in this paper, a low-cost localization system based on RFID technology is considered to monitor the positions of elderly people. In addition, the time-sensitive alert marquee, email, and push notification (Google Cloud Messaging, GCM) ${ }^{(9)}$ functions are used to give an alarm for elderly people who stay too long in the bathroom or other locations outside. The RFID positioning system with the text marquee and GCM push notification is used to identify and monitor elderly people, in addition to analyzing the danger posed to elderly people, by setting up a time-sensitive alert function for elderly people in potentially dangerous locations. On the other hand, the fuzzy logic developed by Zadeh ${ }^{(10)}$ and Huang et al. ${ }^{(1)}$ has attracted considerable attention because of its universal approximation ability in the nonlinear problem. ${ }^{(12)}$ Moreover, in this study, the fuzzy logic is applied to the RFID-based localization system to increase the positioning accuracy of elderly people. This system aims to improve the effectiveness of elderly care.

The remainder of this paper is organized as follows. In Sect. 2, we present the system structure and methods. In Sect. 3, we describe the experimental results of the RFID position monitoring system. In Sect. 4, we discuss the results. In Sect. 5, we present our conclusions.

\section{System Structure and Methods}

In this study, an RFID-based position-monitoring system is proposed, which consists of an integrated client side, a server side, and a sensor side, as shown in Fig. 1. On the sensor side, a reader, tags, and locators use standard RFID communication to transmit messages. All individuals whom the caregivers wish to monitor wear the tag, which sends packets to a backend reader. Additionally, tags can receive position packets sent by the locator. After entering the tag location into the packet, it returns the packet to the backend reader. The reader will receive

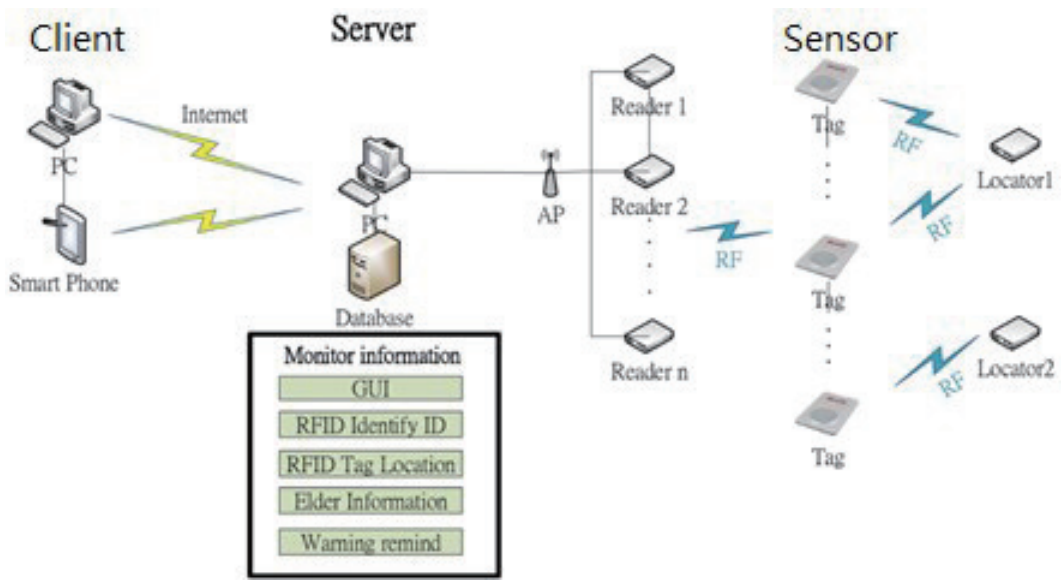

Fig. 1. (Color online) RFID-based monitoring system. 
the packet information sent by the tags at regular intervals; this information includes the tag ID, locator position, and emergency notifications.

The processing center of the system is at the server end, and it is composed of a database and middleware. The bridge between the database and the sensor side is formed of the middleware and wireless base station communication systems. The main function of the middleware is to receive packets returned by the tags worn by the elderly people, analyze the packet data, and store them in the database. The wireless base station or access point (AP) provides a means for communication between mobile devices in the monitoring system. The AP can also provide the staff with a means of monitoring, system management, and data acquisition via a desktop computer or handheld device.

On the client end, caregivers can use the webpage or smartphone application to check the daily attendance of the elderly people, as well as their current status and location. On the equipment side, readers are provided with an external IP. Through external IP addresses on the same network segment, packet information can be returned to the middleware after being received by the AP. Using the network access translator installed on the AP, information can be transferred to the middleware of a designated port. Our system also includes a web version interface to provide a convenient method for caregivers and family members to check the status of an elderly person. The web interface can include a historical record because packet data will be stored in the database.

In addition, the text marquee and GCM push notification are added on the website monitoring system. GCM is a free service that can allow staff to send messages on a variety of platforms (Android, iOS, and Chrome). For example, a server sends messages directly to a single device or a group of devices, and the text marquee is used on the web page screen with dynamic updates. When an elderly person is outside the control area for a long period of time, the marquee will be immediately updated. A large screen for monitoring will allow easy discovery when an elderly person leaves the indoor area.

When elderly people arrive at the senior center every morning, caregivers assist in activating their RFID tags, which send data packets to a server simultaneously. The server deals with packets via programs and shows the statuses and positions of the elderly people at the senior center on a website page. Moreover, a GCM push notification is sent to caregivers' cell phones and a text marquee appears on the website page to alert the staff regarding a potential danger when an elderly person stays in the restroom or outdoors for a long time.

To increase the positioning accuracy of elderly people, fuzzy logic is applied to the RFIDbased localization system. The fuzzy logic developed by Zadeh has attracted considerable attention because of its universal approximation ability in the nonlinear problem. We can establish fuzzy rules by matching input-output pairs through an adaptation procedure. Here, for practical implementation, we establish both membership functions (MBFs) and rules using the heuristic basis and knowledge about input-output relationships. In this study, the outdoor activity of elderly people is considered a fuzzy event and the fuzzy control rule is defined by a three-input and one-output fuzzy system.

To find the optimal possibility of outdoor activity of the $k$ th elderly person, we investigated the $n$th event for the $k$ th elderly person with the parameters $T_{C, k, n}$ for the duration of staying in 
the corridor, $T_{\mathrm{R}, k, n}$ for staying in the restroom, and $T_{p, k, n}$ for a time period in the morning, noon, or afternoon.

We choose two and three triangular MBFs to cover the entire universe of discourse of three inputs, $T_{C, k, n}, T_{R, k, n}$, and $T_{p, k, n}$, and one output, $p_{O A, k, n}$. The two linguistic terms, short (S) and long (L), are chosen to cover the universe of discourse for $T_{C, k, n}$ and $T_{R, k, n}$. The three terms, morning $(\mathrm{M})$, noon $(\mathrm{N})$, and afternoon $(\mathrm{A})$, are chosen to cover the universe of discourse for $T_{p, k, n}$. As for the terms of the outdoor activity, $p_{O A, k, n}$, the three terms, impossible (IP), possible (P), and very possible (VP), are chosen to cover its universe of discourse. The MBFs of the three inputs, $T_{C, k, n}, T_{R, k, n}$, and $T_{p, k, n}$, and one output, $p_{O A, k, n}$, are illustrated in Figs. 2(a)-2(c).

In this paper, we consider the fuzzy control rules of a two-input, single-output fuzzy system to be

$$
R^{j}: \text { IF } T_{C, k, n} \text { is } F_{1}^{l_{1}} \text { AND } T_{R, k, n} \text { is } F_{2}^{l_{2}} \text { AND } T_{p, k, n} \text {, is } F_{3}^{l_{3}} \text {, THEN } p_{O A, k, n}=F_{4}^{l_{4}}
$$

where $F_{1}^{l_{1}}, F_{2}^{l_{2}}, F_{3}^{l_{3}}$, and $F_{4}^{l_{4}}$ are the linguistic terms of the input variables $T_{C, k, n}, T_{R, k, n}$, and $T_{p, k, n}$ and the output variable $p_{O A, k, n}$, respectively, and $l_{1}, l_{2}=1,2, l_{3}, l_{4}=1,2,3$, and the index of rule $j=1,2, \ldots, 12$. The rule base, shown in Table 1, including 12 fuzzy IF-THEN rules, can be established heuristically from the experimental results, whereby the relationships between the possible outdoor activity of the elderly and their durations along corridors and in the restroom can be inferred.

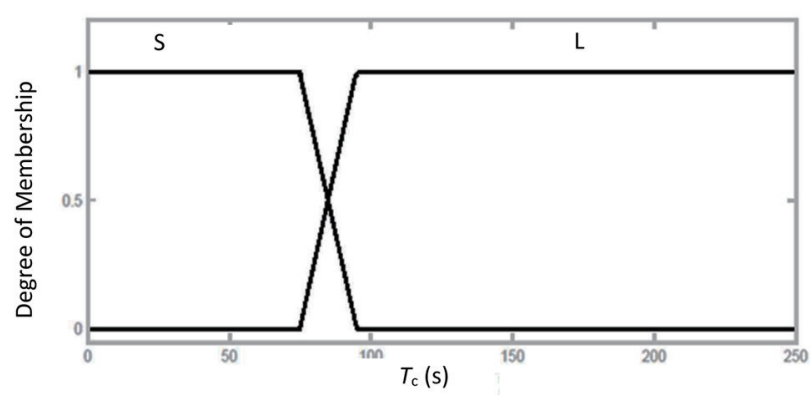

(a)

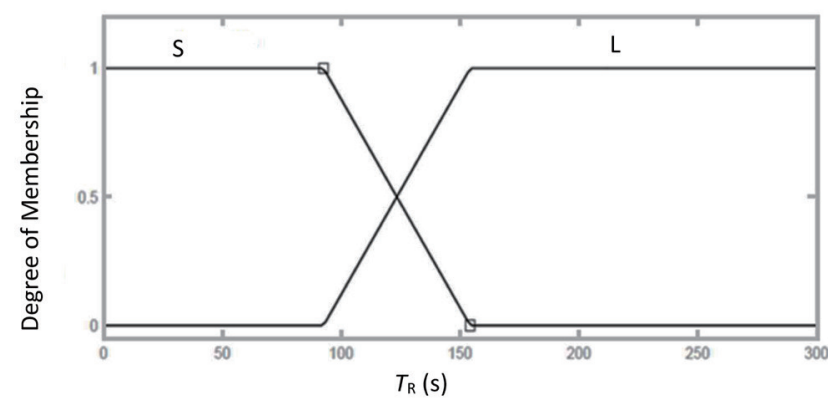

(b)

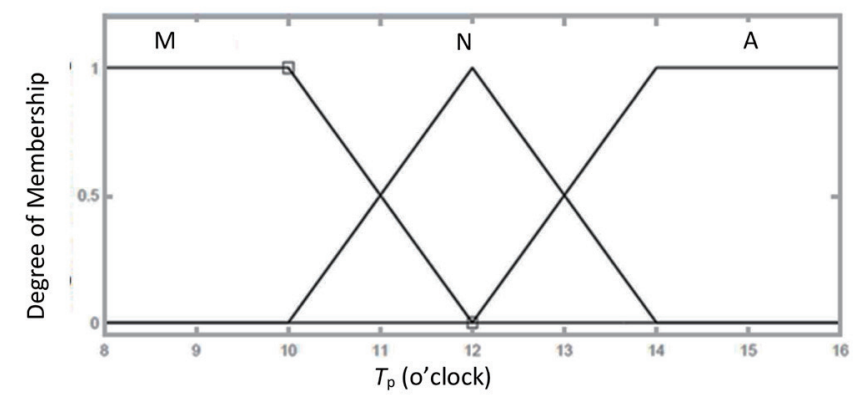

(c)

Fig. 2. Membership functions of FIS of outdoor activity prediction: (a) duration of staying in the corridor, $T_{C}$, (b) duration of staying in the restroom, $T_{R}$, and (c) time of activity event, $T_{p}$. 
Table 1

Rule base of FIS.

\begin{tabular}{lccccccc}
\hline \multirow{2}{*}{$T_{p}$} & $T_{C}$ & \multicolumn{2}{c}{$\mathrm{S}$} & & \multicolumn{2}{c}{$\mathrm{L}$} \\
\cline { 2 - 3 } \cline { 6 - 7 } & $T_{R}$ & $\mathrm{~S}$ & $\mathrm{~L}$ & & $\mathrm{~L}$ & $\mathrm{~S}$ \\
\hline $\mathrm{M}$ & & IP & IP & & IP & IP \\
$\mathrm{N}$ & & IP & IP & & P & P \\
$\mathrm{A}$ & & VP & IP & & P & VP \\
\hline
\end{tabular}

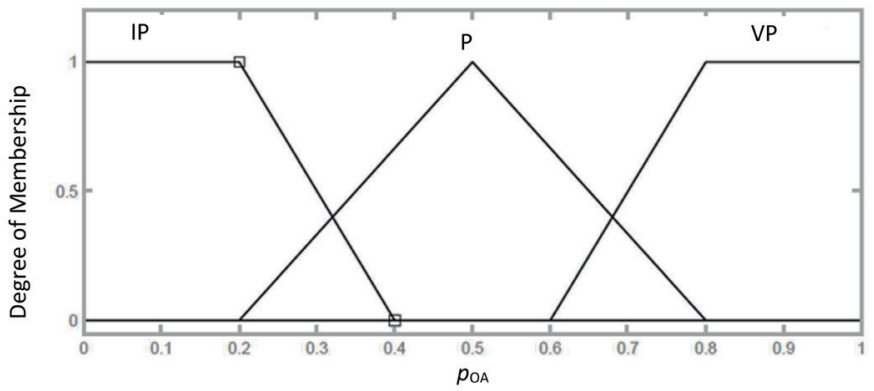

Fig. 3. Membership functions of FIS of outdoor activity prediction: output variable $p_{O A}$.

There are many defuzzification methods available ${ }^{(12)}$ and the following centroid calculation is employed to return the center of the area under the aggregated MBFs curve.

$$
p_{O A, k, n}=\frac{\sum_{i=1}^{q} z_{i} \mu_{F_{4}^{l}}\left(z_{i}\right)}{\sum_{i=1}^{q} \mu_{F_{4}^{l}}\left(z_{i}\right)}
$$

Here, $q$ is the number of quantization levels of the output area under the aggregated MBFs, $z_{i}$ is the amount of inference output at the quantization level $i$, and $\mu_{F_{4}^{l}}\left(z_{i}\right)$ is its membership value in the output fuzzy set $F_{4}^{l}$. Thus, the fuzzy inference system (FIS) mechanism for the prediction of the outdoor activity scheme can be elucidated.

We consider fuzzy logic in this study to assist outdoor activity predictions. Figures 2(a)2(c) show the membership functions of the inputs $T_{C}, T_{R}$, and $T_{p}$, respectively. Moreover, the membership function of the FIS of outdoor activity prediction, output variable $p_{O A}$, is shown in Fig. 3.

\section{Experimental Results}

In this study, the senior center in Chaoyang University of Technology served as the headquarters for developing and implementing the proposed RFID position-monitoring system. The map of the senior center is shown in Fig. 4, where A is an activity room for the elderly, B is the corridor, and $\mathrm{C}$ is the area of bathrooms (restrooms). Two RFID readers are placed in areas A and C. Then, three RFID locators are installed in the activity room, men's bathroom, and women's bathroom. One server is placed in the activity room to collect and analyze the information from the RFID readers; it is connected with a monitor to show the website page for the caregivers.

Figure 5 shows that we can successfully present the current status and locations of the elderly people on the website monitoring page. The blue rectangle indicates the absence of the elderly person. When an elderly person is in the bathroom, the website page shows a yellow 


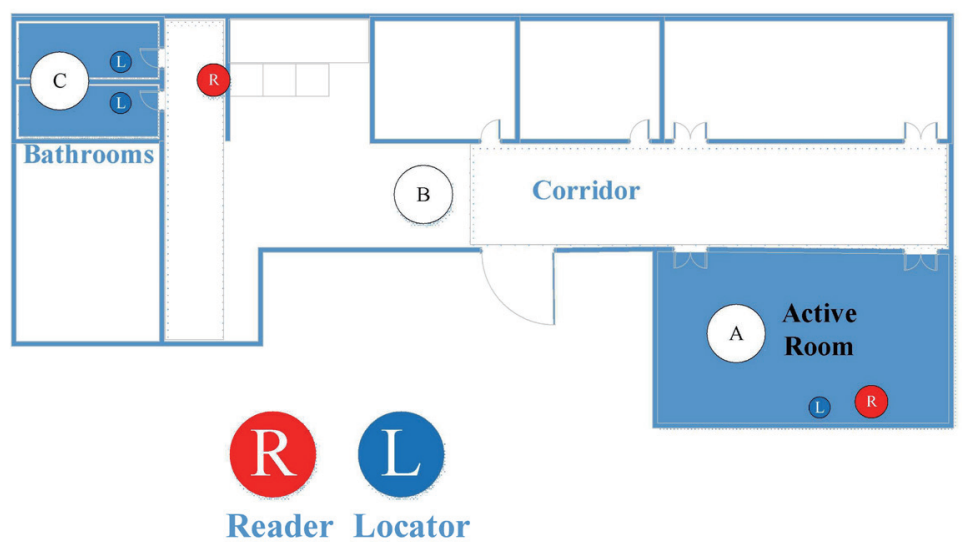

Fig. 4. (Color online) Map of senior center.

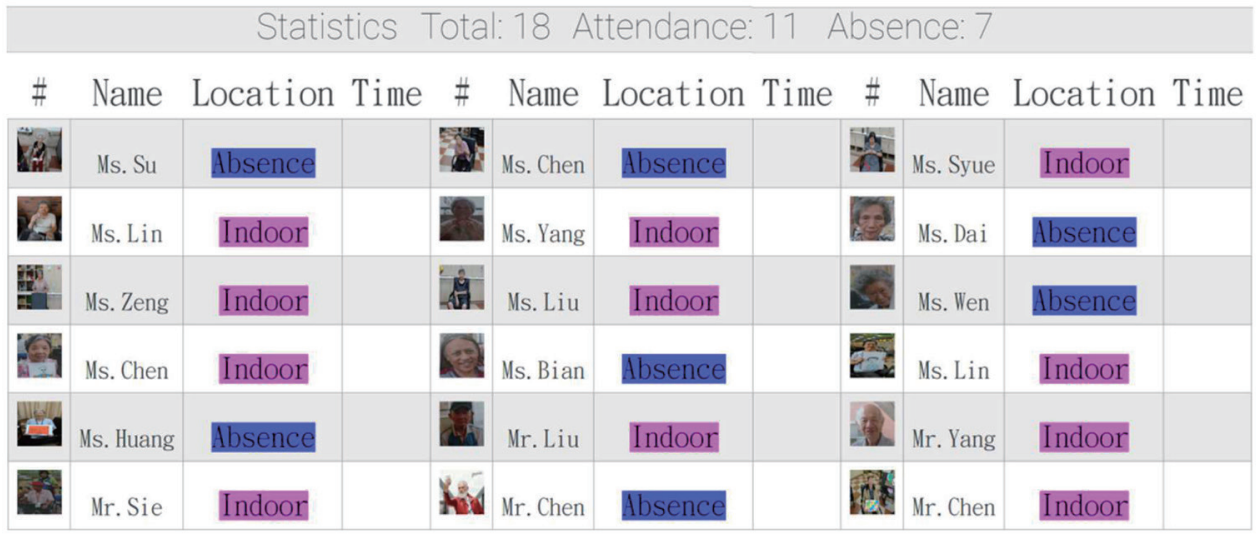

Fig. 5. (Color online) Website monitoring page.

rectangle with the staying time. When they stay outside of the senior center, the red rectangle is presented with the staying time. The pink rectangle is used to present their attendance and indoor status.

Figure 6(a) shows that a text marquee in red immediately appears on the upper left of the website monitoring page if an elderly person spends a longer time than usual in the restroom or outdoors. Simultaneously, the monitoring system sends an email and a GCM push notification to alert staff members regarding the potential danger, as shown in Fig. 6(b). Because elderly people tend to walk at different speeds and use the restrooms for varying durations, caregivers can use the webpage to customize the time limit for each elderly person.

\section{Discussion}

With fuzzy logic added to the RFID-based localization system, we conducted some experiments on localization with elderly people in the senior center, as shown in Table 2. The results show the event predictions of daily actives for elderly people in the senior center. However, a few problems exist concerning the accurate estimation of the locations of the elderly 


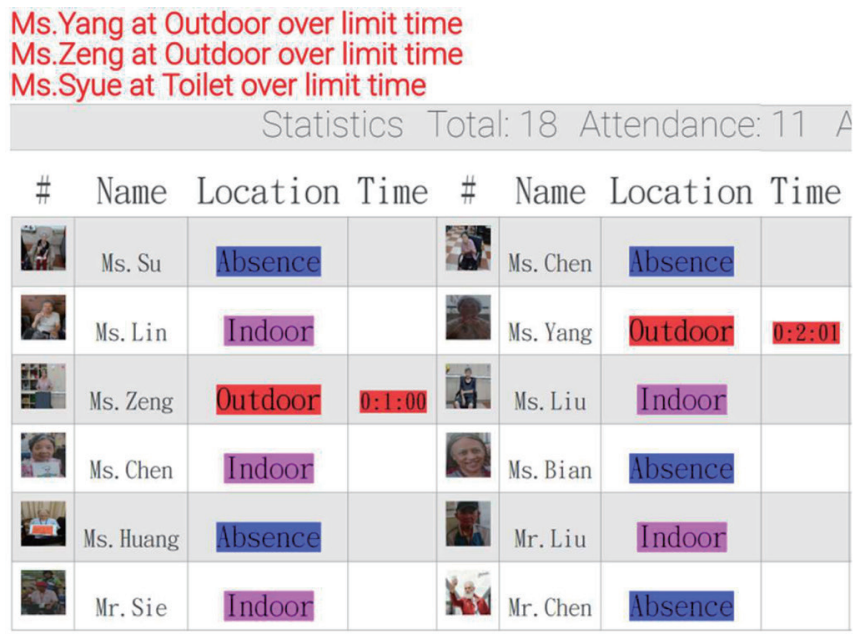

(a)

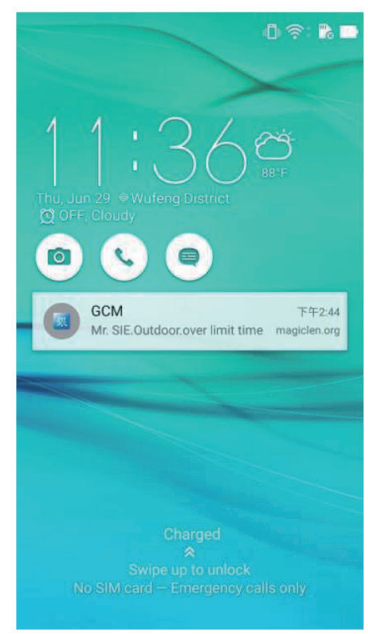

(b)

Fig. 6. (Color online) (a) Text marquee on website monitoring page and (b) GCM push notification.

Table 2

Results obtained by FIS to view events for elderly people.

\begin{tabular}{cccccc}
\hline No. of events & $T_{C}(\mathrm{~s})$ & $T_{R}(\mathrm{~s})$ & $T p$ (o'clock) & $p_{O A}$ & Statuses \\
\hline 1 & 72 & 145 & $11-12$ & 0.168 & Go to Bathroom \\
2 & 81 & 107 & $11-12$ & 0.273 & Go to Bathroom \\
3 & 88 & 104 & $11-12$ & 0.316 & Go to Bathroom \\
4 & 82 & 166 & $15-16$ & 0.314 & Go to Bathroom \\
5 & 74 & 90 & $10-11$ & 0.174 & Go to Bathroom \\
6 & 68 & 0 & $12-13$ & 0.156 & Walk along the Corridor \\
7 & 23 & 0 & $12-13$ & 0.156 & Walk along the Corridor \\
8 & 96 & 0 & $13-14$ & 0.239 & Walk along the Corridor \\
9 & 165 & 0 & $14-15$ & 0.847 & Outdoor Activity \\
10 & 152 & 145 & $14-15$ & 0.54 & Outdoor Activity \\
\hline
\end{tabular}

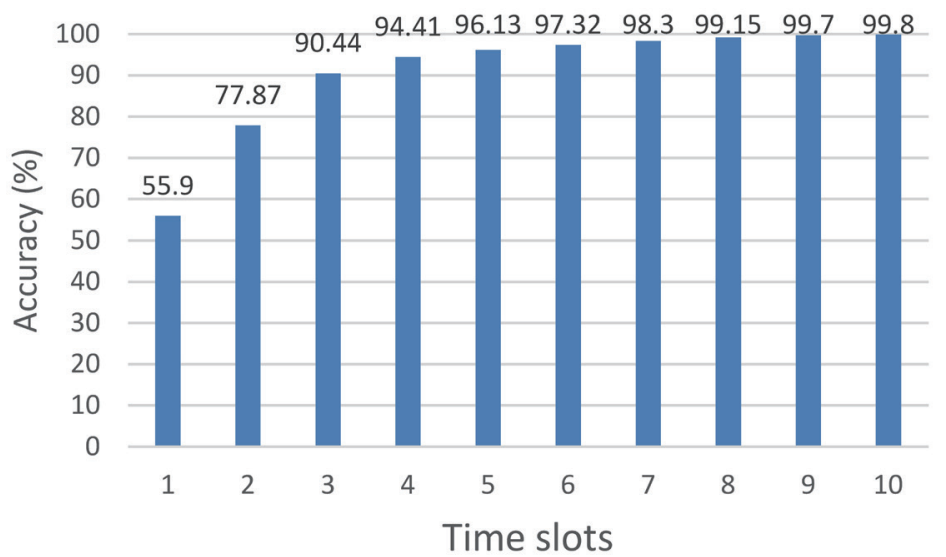

Fig. 7. (Color online) Accuracy of localization.

subjects. Therefore, more data must be collected and analyzed to increase the localization accuracy, as shown in Fig. 7, where the $y$-axis is the localization accuracy (\%) and the $x$-axis is the number of time slots. As the numbers of time slots increase, the accuracy also increases. 


\section{Conclusions}

The design and implementation of the RFID position-monitoring system was presented in this paper with the goal of supporting overworked caregivers on whom the elderly depend on a daily basis. On the basis of the decision-making abilities of the system, caregivers will be alerted to the potential danger when an elderly person spends an unusually long time in a potentially hazardous area, providing caregivers the opportunity to provide assistance and avoid serious accidents. The experimental results showed that the proposed monitoring system can be effective for monitoring the current status of elderly people as they go about their daily lives, and therefore has definite practical functionality. To increase the efficiency and quality of care, fuzzy logic was added to the proposed monitoring system. Then, the results showed that caregivers can determine the locations and time of outdoor activities of elderly people in various areas more accurately by collecting and analyzing data through fuzzy logic.

\section{Acknowledgments}

This work was supported in part by the Ministry of Science and Technology of Taiwan under grant numbers MOST 106-2218-E-027-017 and MOST 106-2221-E-324-020.

\section{References}

1 R. Bhilare, S. Mali, and E. Dutkiewicz: Proc. IEEE India Conf. (2015) 1.

L. Zhang, J. Liu, and H. Jiang: Proc. IEEE Sensors (2012) 1.

3 S. H. Fang, C. H. Wang, T. Y. Huang, C. H. Yang, and Y. S. Chen: IEEE Commun. Lett. 16 (2012) 564.

4 M. Collotta and G. Pau: IEEE J. Sel. Areas Commun. 33 (2015) 2988.

5 P. Bahl and V. N. Padmanabhan: Proc. IEEE INFOCOM 2 (2000) 775.

6 M. Meints: http://www.fidis.net/resources/fidis-deliverables/hightechid/int-d3700/ (accessed August 2016).

7 R. Want, A. Hopper, V. Falcao, and J. Gibbons: ACM Trans. Inf. Syst. 40 (1992) 91.

8 L. M. Ni, Y. Liu, Y. C. Lau, and A. P. Patil: Proc. IEEE Inter. Conf. Pervasive Computing and Communications (2003) 407.

9 Y. S. Yilmaz, B. I. Aydin, and M. Demirbas: Proc. IEEE Globecom (2014) 2807.

10 L. A. Zadeh: Inf. Control 8 (1965) 338.

11 Y.-F. Huang, N.-C. Wang, T.-H. Tan, and C.-P. Tsai: Inter. J. Fuzzy Sys. 10 (2008) 231.

12 C.-T. Lin and C. S. G. Lee: Neural Fuzzy Systems: A Neural-Fuzzy Synergism to Intelligent Systems (Prentice-Hall, New Jersey, 1996).

\section{About the Authors}

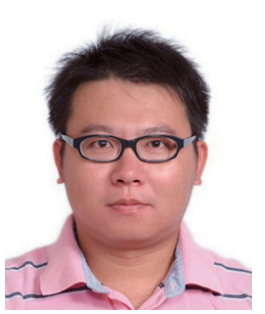

Chuan-Bi Lin received his B.S. degree from Tatung University, Taiwan, in 1994 and his M.S. degree from the University of Bridgeport, USA, in 2001. He received his Ph.D. degree in electrical and computer engineering from New Jersey Institute of Technology, Newark, USA, in 2008. He was a postdoctoral researcher with the Department of Electrical and Computer Engineering, New Jersey Institute of Technology, from 2008 to 2009. Since 2009, he has been an assistant professor in the Department of Information and Communication Engineering at Chaoyang University of Technology. His research interests are in computer networks, switching and routing, softwaredefined networks (SDNs), and applications of IoT. Currently, he is focused on the Industry-Academia cooperation projects of IoT. 


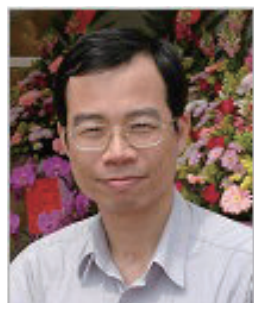

Yung-Fa Huang received his B.S. degree in electrical engineering from National Taipei University of Technology, Taipei, in 1982, M.S. degree in electrical engineering from National Tsing Hua University, Hsinchu, Taiwan, in 1987, and Ph.D. degree in electrical engineering from National Chung Cheng University, Chiayi, Taiwan, in 2002. From 1982 to 1984, he fulfilled his military service for Taiwan in the Air Force. From 1987 to 2002, he was an instructor in Chung Chou Institute of Technology, Yuanlin, Taiwan. From February 2002 to July 2004, he was with the Department of Electrical Engineering, Chung Chou Institute of Technology, as an associate professor. From August 2004 to July 2007, he was an associate professor in the Graduate Institute of Networking and Communication Engineering, Chaoyang University of Technology, Taichung, Taiwan. From August 2007 to July 2008, he was the Head of the Department of Computer and Communication Engineering and the Chair of the Graduate Institute of Networking and Communication Engineering, Chaoyang University of Technology. From August 2008 to July 2010, he was the Head of the Department of Information and Communication Engineering, Chaoyang University of Technology. Since September 2012, he has been a professor at the Department of Information and Communication Engineering, Chaoyang University of Technology. His current research interests include multiuser detection in OFDM-CDMA cellular mobile communication systems, communication signal processing, fuzzy systems, and wireless sensor networks. He is a member of IEEE and serves as a co-chair of the IEEE SMC Society Technical Committee on Intelligent Internet Systems.

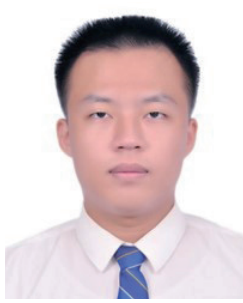

Long-Xin Chen is a graduate student in the Department of Information and Communication Engineering at Chaoyang University of Technology. $\mathrm{He}$ received his B.S. degree in information and communication engineering from Chaoyang University of Technology. His research interest is in computer networks, software-defined networks, and applications of Internet of Things (IoT).

Jian-Ming Hong received his B.S. and M.S. degrees in information and communication engineering from Chaoyang University of Technology in 2014 and 2016, respectively. His research focused on computer networks, control systems with high-speed and real-time communication links, and applications of Internet of Things (IoT). He is looking for a job to become an engineer. 